\title{
Unstable relics and the rescue of pure Cold Dark Matter models
}

\section{Montanino *}

SISSA/ISAS, Via Beirut 4, I-34014 Trieste, Italy

E-mail: montanin@sissa.it!

ABstract: Pure CDM models give too much power to the spectrum of density fluctuations at small scales. This problem can be solved if the horizon length at the matter-radiation equivalence is increased. One solution is to delay the equivalence time through the injection of non thermal radiation coming from the decay of an unstable relic particle. We show how several realistic particle models can be good candidates to accomplish this task.

\section{Introduction}

Standard Cold Dark Matter (CDM) models, characterized by a flat universe with cold particles as dark matter, turned out quite successful and its predictions about the structure formation of galaxies and clusters of galaxies agreed well with

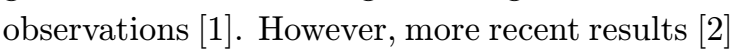
showed that, once normalized at large scale by COBE data, the standard CDM model predicts more power than observed at small scale. To be more quantitative, the spectrum of density fluctuations in models of pure CDM as function of the scale $\lambda$ of observation is given by

$$
\left(\frac{\delta \rho}{\rho}\right) \propto \frac{\lambda^{\prime-2}}{1+1.7 \frac{l}{\lambda^{\prime}}+9\left(\frac{l}{\lambda^{\prime}}\right)^{3 / 2}+\left(\frac{l}{\lambda^{\prime}}\right)^{2}},
$$

where $\lambda^{\prime}=\lambda / h^{-1}\left(h=H_{0} / 100 \mathrm{Km} \mathrm{Mpc}^{-1} \mathrm{sec}^{-1}\right)$ and $l=\left(\Omega_{\mathrm{M}} h\right)^{-1}$. Here $\Omega_{\mathrm{M}}=\Omega_{\mathrm{BM}}+\Omega_{\mathrm{CDM}} \simeq$ $\Omega_{\mathrm{CDM}}$ - where BM stands for "Baryonic Matter" - since nucleosynthesis requires $\Omega_{\mathrm{BM}} \leq 0.1$

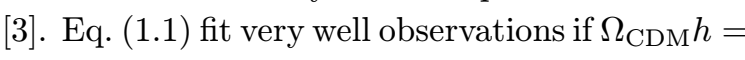
$0.2 \div 0.3$ (see e.g. Fig. 1 in [핑)

Inflationary theories predict $\Omega_{\text {tot }}=1$. This result seem confirmed by the observation of the first acoustic peak of of the Cosmic Background Radiation (CBR) [i5in]. On the other hand, recent observations suggest $h=0.67 \pm 0.10$ [i] any case a value of $\Omega h \leq 0.4$ seems to be ex-

${ }^{*}$ Co-authors: A. Masiero and M. Peloso cluded. This strongly disfavor models with pure CDM (i.e., with $\Omega_{\mathrm{CDM}}=1$ ). The simplest way to solve this inconsistency is to postulate that the CDM is only a part of the dark energy of the universe: $\Omega_{\mathrm{CDM}}<\Omega_{\mathrm{tot}}=1$. This is possible in models with mixed hot (or "warm") plus cold dark matter, or in models with a non-zero cosmological constant. The latter are the most appealing at the moment because the incoming evidence for an accelerating universe from far supernovae measurements [i]

We examine a different way to solve this puzzle. We study the possibility that, in addition to the CDM, there exist decaying (or sometimes called volatile) particles. The relativistic decay products of these particles can increase the horizon length at the equivalence time. As we see, in this way it is possible to increase the power

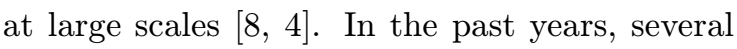
models which implement this idea have been pro-

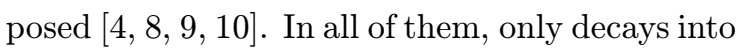
weakly interacting particles (as gravitinos or neutrinos) were considered, since very stringent cosmological and astrophysical bounds apply when more interacting particles are produced. However, popular extensions of the Standard Model (and in particular supersymmetric models) provide several other natural candidates both for the CDM and for massive and unstable particles that we need as the volatile component. In [1] we up- 
dated and extended the analyses proposed so far, showing in most cases the difficulties that prevent the realization of the scheme, but also pointing out viable models in a few examples. In this talk we briefly review the results of this work.

\section{The general model}

We start noticing that during radiation dominated era(s) sub-horizon fluctuations are frozen while super-horizon ones grow. Let $\lambda_{\text {eq }}$ the scale corresponding to the horizon at the epoch of matterradiation equivalence. In the standard Big Bang cosmology, from the measure of CBR energy density and assuming three neutrinos species is easy to obtain [īi

$$
\lambda_{\mathrm{eq}}^{\prime}=30 \mathrm{Mpc}\left(\Omega_{\mathrm{CDM}} h\right)^{-1} .
$$

Starting from a primordial Zel'dovich (scale invariant) spectrum, one can say that for scales $\lambda<\lambda_{\text {eq }}$ the fluctuations are roughly constant while for $\lambda>\lambda_{\text {eq }} \delta \rho / \rho \propto\left(\lambda / \lambda_{\text {eq }}\right)^{-2}$ [the exact form is given by Eq. (11.1)]. From this relation we see that delaying matter domination (and thus increasing $\lambda_{\text {eq }}$ ), we give more power to larger scales respect to the smaller. The best fit with the data is obtained for $\lambda_{\text {eq }}^{\prime}=150 \mathrm{Mpc}$ (i.e., for $\Omega_{\mathrm{CDM}} h=0.2$ ).

Instead of lowering $\Omega_{\mathrm{CDM}} h$ a raising of $\lambda_{\text {eq }}$ can be achieved by considering an unstable $X$ particle which decays into relativistic debris [it, [1]. This particles survives for a time $\tau_{X} \gg 1 \mathrm{sec}$ (the time of neutrino decoupling) and dominate the universe for a short period after that they decay (for simplicity we use the approximation of simultaneous decay). Then, the decay products give rise to a second period of radiation domination. In Fig. 高 [1] resentation of this idea. Two remarks are in oder:

1. The decay products cannot be photons alone. In fact, if $\tau_{X}<10^{6} \mathrm{sec}$ (the "recombination time") photons would thermalize and just contribute to the CBR energy density we measure today from which we infer Eq. (2.1). On the other hand, there are strong limits for non-thermal diffuse photons [3] , thus excluding a radiative decay with $\tau_{X}>10^{6} \mathrm{sec}$.

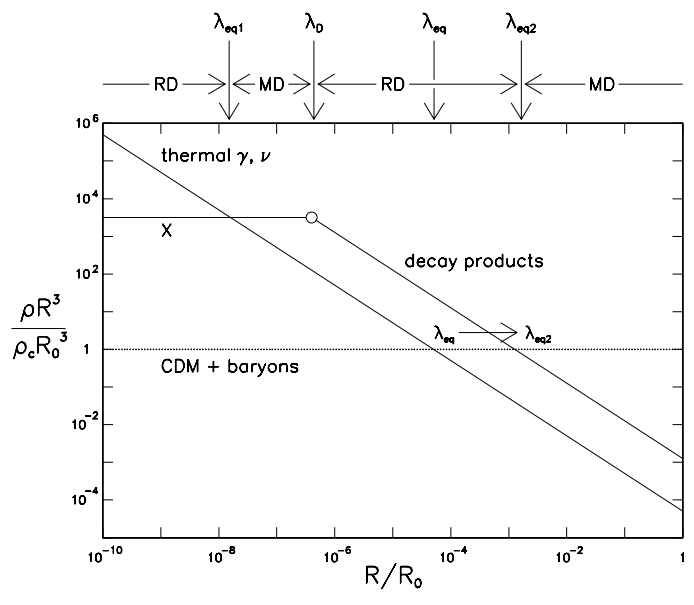

Figure 1: Schematic representation of the model.

2. If $X$ would decay in charged particles these immediately comptonize the background photons and generate an electromagnetic cascade. The decay into charged particles is thus equivalent to the decay into photons.

This considerations imply that the decay products must be partially or totally "invisible", i.e., weak interacting particles such as neutrinos, ${ }^{1}$ light uncharged Supersymmetric (SUSY) particles, axions, massless scalars, etc. Since these particles are decoupled at the decay time, they are out from thermal equilibrium.

From Fig. (1) we see that the shift of the equivalence scale from $\lambda_{\text {eq }}$ to $\lambda_{\text {eq } 2}>\lambda_{\text {eq }}$ is thus possible. The following relation holds [1] [1]

$$
\frac{\lambda_{\mathrm{eq} 2}}{\lambda_{\mathrm{eq}}} \simeq\left[1.5\left(\frac{\tau_{X}}{\mathrm{sec}}\right)^{2 / 3}\left(\frac{m_{X} Y_{X}^{i}}{\mathrm{MeV}}\right)^{4 / 3}+1\right]^{1 / 2}
$$

Here $m_{X}$ and $Y_{X}$ are respectively the mass and the comoving number density (i.e., the number density divided by the entropy density of photons, $s \simeq 1.8 g_{s *} n_{\gamma}$ ) of the particle $X$ and the superscript $i$ indicates that we must consider only the fraction of energy which goes into invisible relativistic particles. From the requirement $\lambda_{\text {eq } 2} \simeq$

\footnotetext{
${ }^{1}$ High energy neutrinos can scatter off background neutrinos with the consequent creation of an electromagnetic shower [12]. For this reason also neutrinos are not completely safe from problems and should be handled with care.
} 
$150 \mathrm{Mpc}$ we get the following relation:

$$
\left(\frac{\tau_{X}}{\mathrm{sec}}\right)\left(\frac{m_{X} Y_{X}^{i}}{\mathrm{MeV}}\right)^{2} \simeq 0.55\left[\left(\frac{h}{0.2}\right)^{2}-1\right]^{3 / 2}
$$

Besides Eq. (2.3i) other cosmological bounds must be satisfied:

- At the time of neutrino decoupling the energy density of the $X$ particles must be $<1$ neutrino species to avoid problems with Big Bang Nucleosynthesys (BBN) [1슨

$$
\frac{m_{X} Y_{X}}{\mathrm{MeV}}<0.1
$$

- The fraction of energy which goes into invisibles must be actually high enough to obtain the first period of matter domination. This condition led to

$$
\frac{m_{X} Y_{X}^{i}}{\mathrm{MeV}}>3.6 \times 10^{-6} h^{2} .
$$

When also radiative decays are involved (i.e., if $X$ particles decay into photons or charged particles) there are additional bounds coming from BBN. In fact high energy photons are dangerous for BBN since they easily destroy the primordial deuterium and dramatically change the relative composition of primordial elements. Stringent bounds on $X$ lifetime and abundance can be fixed by the observations [14 4]. Combining Eqs. (2.3i) and $([-2.4)$ with the bounds in [1 14$]$ one can see that decays in which a non-negligible fraction of the energy goes into "visible" are strongly constrained. In particular the lifetime must be limited to the interval (see Fig. 2 in [i $\left.\overline{1}_{1}^{1}\right) 10^{3} \leq$ $\tau_{X} / \sec \leq 10^{4}$. With this limit the bound (2. always satisfied [1] 1']

In the following section we briefly examine three realistic particle models that can play the job described above.

\section{Three particles models}

\subsection{SUSY with broken $R$ parity}

Let us stick now in the Minimal Supersymmetric extension of the Standard Model (MSSM) and suppose for simplicity the bino (i.e., the superpartner of the $\mathrm{U}(1)_{Y}$ gauge boson) as Lightest
Supersymmetric Particle (LSP). If $R$-parity is conserved, the LSP is stable. Let us suppose that a small quantity of R-parity violation is allowed through the term $\lambda L L E^{c}$ in the superpotential. The decay

$$
\widetilde{B} \rightarrow \nu e^{+} e^{-}
$$

is thus allowed through sneutrino or selectron exchange. In this case $\widetilde{B}$ can play the role of the $X$ particle. (If binos are stable they are a good candidate for CDM; obviously, in our case we need of a different particle as source of CDM.) Since the electrons comptonize the background photons, they are the visible part of the decay and all the considerations about radiative decays apply. The neutrinos are the invisible part and they carry out about $1 / 3$ of the initial energy.

The bino lifetime is given by [1]

$$
\tau_{\tilde{B}}^{-1}=\lambda^{2} \frac{\alpha_{\text {e.m. }} . y_{\tilde{f}_{R}}^{2}}{192(2 \pi)^{2} \cos ^{2} \theta_{W}} \frac{m_{\tilde{B}}^{5}}{m_{\tilde{f}}^{4}},
$$

where $m_{\tilde{f}}$ is the mass of the sfermion (neutrino or selectron) and $y_{\tilde{f}_{R}}$ its hypercharge. Moreover the bino abundance is given by [1] $\left[\sigma^{\prime}\right]$

$$
Y_{\tilde{B}} \simeq 4 \times 10^{-9}\left(\frac{m_{\tilde{f}}}{\mathrm{TeV}}\right)^{4}\left(\frac{10^{2} \mathrm{GeV}}{m_{\tilde{B}}}\right)^{3} .
$$

We suppose for simplicity $m_{\tilde{\nu}} \gg m_{\tilde{e}}$ so that selectron exchange prevail and we can substitute in Eqs. (3.1) and (3.2. hypercharge to $m_{\tilde{f}}$ and $y_{f_{R}}$.

Combining Eqs. (3.1) and (3.2i) with Eq. (2.3i) and choosing the bino mass and lifetime as free parameters, we obtain, for $h=0.5$ (as a numerical example) [i] $\left.i_{1}^{1}\right]$

$$
\begin{aligned}
\frac{m_{\tilde{e}}}{\mathrm{TeV}} & \simeq 5.0\left(\frac{m_{\tilde{B}}}{10^{2} \mathrm{GeV}}\right)^{1 / 2}\left(\frac{10^{3} \mathrm{sec}}{\tau_{\tilde{B}}}\right)^{1 / 8} \\
\frac{\lambda}{10^{-10}} & \simeq 1.5\left(\frac{m_{\tilde{B}}}{10^{2} \mathrm{GeV}}\right)^{3 / 2}\left(\frac{\tau_{\tilde{B}}}{10^{3} \mathrm{sec}}\right)^{3 / 4}
\end{aligned}
$$

As we can see, for realistic values of the bino mass and lifetimes both the selectron mass and the R-breaking coupling are quite realistic. In particular, the value of $\lambda$ is well below the experimental bounds $\left(\lambda \leq 10^{-2}[17]\right)$.

\subsection{SUSY Peccei-Quinn symmetry break- ing}

The second possibility we consider is the SUSY extension of the Peccei-Quinn symmetry, invoked 
to solve the strong CP problem [1] that the superpartner of the axion, the axino, is the LSP and the photino is the NLSP. In this hypothesis the decay ${ }^{2}$

$$
\widetilde{\gamma} \rightarrow \widetilde{a} \gamma
$$

is allowed through a fermion-sfermion loop (notice that in this case we suppose that the R-parity is conserved). In his scheme the $\widetilde{\gamma}$ play the role of the $X$ particles, the photons are the visible energy while the axinos are the invisible one. As obvious, in order the axinos to be relativistic we must have $m_{\tilde{\gamma}} \gg m_{\tilde{a}}$. This requirement will be verified a posteriori. In this limit, axinos carry about $1 / 2$ of the initial axino energy.

Supposing that the sfermion masses are approximatively equal, the top-stop loop prevail on the other fermions-sfermion loops because the high mass of the top quark respect to the other fermions. The photino lifetime is hence given by $\left[19^{\prime}\right]$

$$
\tau_{\tilde{\gamma}}^{-1}=\frac{\alpha_{\text {e.m. }}^{2}}{18 \pi^{3} V_{\mathrm{PQ}}^{2}} m_{\tilde{\gamma}}^{3}\left[f\left(m_{t}^{2} / m_{\tilde{t}}^{2}\right)\right]^{2},
$$

where $V_{\mathrm{PQ}}$ is the Peccei-Quinn breaking scale, $m_{t}\left(m_{\tilde{t}}\right)$ is the top (stop) mass and

$$
f(x)=\frac{x}{1-x}\left[1+\frac{\log x}{1-x}\right] .
$$

The photino abundance is given by [1] [6] $_{1}^{\prime}$

$$
Y_{\tilde{\gamma}} \simeq 5 \times 10^{-5}\left(\frac{m_{\tilde{t}}}{\mathrm{TeV}}\right)^{4}\left(\frac{10^{2} \mathrm{GeV}}{m_{\tilde{\gamma}}}\right)^{3} .
$$

Following the notation of [20 $\left.{ }_{0}^{-}\right]$, we consider type $I I$ (i.e., light) axinos, whose mass is given by

$$
\frac{m_{\tilde{a}}}{\mathrm{keV}} \simeq\left(\frac{V_{\mathrm{PQ}}}{10^{12} \mathrm{GeV}}\right)^{-1}
$$

Again, sticking Eqs. (3.5i), (3.6i), and (3.7i) into

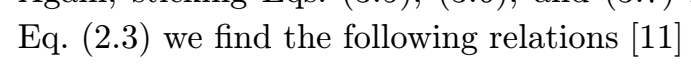

$$
\begin{aligned}
\frac{m_{\tilde{t}}}{\mathrm{TeV}} & \simeq 1.1\left(\frac{m_{\tilde{\gamma}}}{10^{2} \mathrm{GeV}}\right)^{1 / 2}\left(\frac{10^{3} \mathrm{sec}}{\tau_{\tilde{\gamma}}}\right)^{1 / 8} \\
\frac{m_{\tilde{a}}}{\mathrm{keV}} & \simeq 1.0\left(\frac{10^{2} \mathrm{GeV}}{m_{\tilde{\gamma}}}\right)^{3 / 2}\left(\frac{10^{3} \mathrm{sec}}{\tau_{\tilde{\gamma}}}\right)^{1 / 2}
\end{aligned}
$$

\footnotetext{
${ }^{2}$ Another possible model already studied in literature is the decay of gravitino into axino plus axion. The reader is addressed to [4] for this scheme.
}

and

$$
\frac{V_{\mathrm{PQ}}}{10^{12} \mathrm{GeV}} \simeq\left(\frac{m_{\tilde{\gamma}}}{10^{2} \mathrm{GeV}}\right)^{3 / 2}\left(\frac{\tau_{\tilde{\gamma}}}{10^{3} \mathrm{sec}}\right)^{1 / 2}
$$

It is possible to check that for $m_{\tilde{\gamma}} \simeq O\left(10^{2} \mathrm{GeV}\right)$ and $m_{\tilde{a}} \simeq O(\mathrm{keV})$ axinos are still relativistic at the equivalence time.

\subsection{Neutrino decay into scalars}

We now suppose a theory in which neutrinos are coupled with massless scalars through an effective Lagrangian of the form

$$
\mathcal{L}_{\nu \phi}=\frac{1}{V} \bar{\nu}^{\prime} \gamma^{\mu} \nu \partial_{\mu} \phi
$$

This Lagrangian can arise from theories with a global symmetry spontaneously broken. In this theories a Goldstone boson (the field $\phi$ ) survive. The value $V$ is of the order of the symmetry breaking scale.

A typical example of this theories is the majoron model [21]. In this model an isosinglet scalar $\phi$ (the majoron) breaks the global lepton number. Neutrinos are coupled both with the majoron and with the ordinary Standard Model Higgs doublet. If $V \simeq\langle\phi\rangle \gg M_{\text {e.w. }}$, where $M_{\text {e.w. }}$. is a typical electroweak scale (e.g., of the order of the mass of the bottom quark), left-handed neutrinos acquire a mass of the order $M_{\text {e.w. }}^{2} / V$ through the See-Saw mechanism [22]. In the majoron model the main bound on the scale $V$, $V>1 \mathrm{TeV}$ comes from majoron emission in supernovae explosions, and in particular from the neutrino pulse observation from SN1987A $[\overline{2} \overline{2} \overline{3}]$. Another model of neutrino coupling with scalars is familon model. Familons are Goldstone bosons arising from the break of an horizontal symmetry. The effective interaction Lagrangian is similar

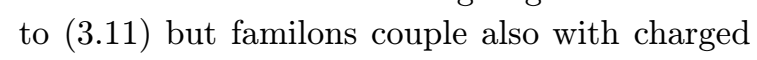
leptons. For this reason there is a very strong limit on $V, V>8 \times 10^{10} \mathrm{GeV}$, coming from energy loss through familon bremsstrahlung of the electrons in stars $[2 \overline{3} \overline{3}]$. This strong bound make the familon model not suitable for our pourposes [1] $11_{1}^{\prime}$. In the next we limit ourself only to majoron model.

In this model the neutrino decay into neutrino plus majoron

$$
\nu^{\prime} \rightarrow \nu \phi
$$


(with $m_{\nu^{\prime}}>m_{\nu}$ ) is possible and the neutrino $\nu^{\prime}$ can play the role of the $X$ particle. Moreover, since in this model the decay products are totally invisible no BBN bounds on lifetime apply. If we suppose $m_{\nu^{\prime}}<1 \mathrm{MeV}$ the neutrino abundance is

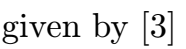

$$
Y_{\nu^{\prime}} \simeq 3.9 \times 10^{-2}
$$

In the limit $m_{\nu^{\prime}} \gg m_{\nu}$ the neutrino lifetime is given by $\left[\underline{9}\left[\begin{array}{l}0 \\ 0\end{array}\right]\right.$

$$
\frac{\tau_{\nu^{\prime}}}{\mathrm{sec}} \simeq 6 \times 10^{9}\left(\frac{V}{10^{8} \mathrm{GeV}}\right)^{2}\left(\frac{\mathrm{keV}}{m_{\nu^{\prime}}}\right)^{3} .
$$

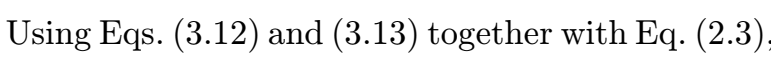
we obtain the following relations [1]

$$
\begin{aligned}
\frac{m_{\nu^{\prime}}}{\mathrm{keV}} & \simeq 0.6\left(\frac{V}{10^{8} \mathrm{GeV}}\right)^{2} \\
\frac{M_{\text {e.w. }}}{\mathrm{GeV}} & \simeq 7.4\left(\frac{V}{10^{8} \mathrm{GeV}}\right)^{3 / 2}
\end{aligned}
$$

and

$$
\frac{\tau_{\nu^{\prime}}}{\mathrm{sec}} \simeq 3.7 \times 10^{10}\left(\frac{10^{8} \mathrm{GeV}}{V}\right)^{4} .
$$

As we see, the only free parameter is the breaking scale $V$. From Eqs. ( $(2 . \overline{4})$ and $(2.51)$ we get

$$
0.2<\frac{V}{10^{8} \mathrm{GeV}}<66 \text {. }
$$

This condition translates into

$$
\begin{aligned}
23 \mathrm{eV} & <m_{\nu^{\prime}}<2.6 \mathrm{MeV} \\
1.9 \times 10^{3} & <\frac{\tau_{\nu^{\prime}}}{\mathrm{sec}}<2.3 \times 10^{13}
\end{aligned}
$$

\section{Discussion and conclusions}

In this talk we have explored the possibility to rescue the pure CDM models by the addition of unstable relic particle decaying (in part or completely) into invisible relativistic products. We have showned three particle models that can accomplish this scheme.

In this talk we have showned only the successful models neglecting the unsuccessful. In

\footnotetext{
${ }^{3}$ It is worth mention that we make use of a non minimal majoron model since in the minimal one the neutrino lifetime would be bigger than the age of the universe [24].
}

particular, we tried with supergravity models with conserved $(\widetilde{g} \rightarrow \nu \widetilde{\nu}, \widetilde{\gamma} \rightarrow \gamma \widetilde{g})$ and broken $(\widetilde{g} \rightarrow$ $\nu \gamma)$ R-parity in the context of Gauge Mediated Supersymmetry breaking [25i] or neutrino decay in three neutrinos in the context of Left-Right symmetric extensions of the Standard Model. We will not discuss here in detail the reason for this failures ${ }^{4}$ but simply state the main problems that make this scheme hard to implement.

The first difficulty is that $\tau_{X}>1 \mathrm{sec}$ is a very long lifetime, hard to obtain in particle physics. The second difficulty is that, for radiative decay, BBN bounds require a quite big $X$ abundance [1]1]. This two requests often force the parameters in the theory to very unnatural values or in disagreement with other observations. For example, in the case of $\widetilde{g} \rightarrow \nu \widetilde{\nu}$ decay, a strong fine-tuning in the difference $m_{\tilde{g}}-m_{\tilde{\nu}}$ is required to assure a gravitino lifetime $>1 \mathrm{sec}$, with the consequence that the energy fraction going into neutrinos is insufficient to provide the equivalence shift. Also in the case of $\widetilde{\gamma} \rightarrow \gamma \widetilde{g}$ decay, a too high value of $m_{\tilde{g}}(>100 \mathrm{MeV})$ is required in order to have a sufficiently long lifetime, with the consequence that gravitinos are no longer relativistic at the equivalence time. Conversely, in the case of $\widetilde{g} \rightarrow \nu \gamma$ decay, a value of R-breaking parameter $\lambda \gg 1$ (thus in disagreement with accelerator limits) is required to obtain a sufficiently short lifetime $\left(\tau<10^{4} \mathrm{sec}\right)$, since in this case one half of the available energy is visible and thus BBN limits apply. Another example is the neutrino radiative decay $\nu^{\prime} \rightarrow \nu \gamma$ : in this case other astrophysical considerations (coming mainly from the observation of supernovae [ij] $)$ completely rule out this channel for our pourposes, independently from the particle model that could allow this decay.

Moreover, also in those models that we have considered and that can perform the shift of equivalence scale, a high degree of fine tuning in the parameters is required. This happens mostly when radiative decay are involved. In fact, in this case the lifetime is confined in a narrow interval $\left(10^{3} \div 10^{4} \mathrm{sec}\right)$. This and other considerations make this scheme very hard to implement, and

\footnotetext{
${ }^{4}$ For a complete discussions about the unsuccessful models see [i11].
} 
works only in a very limited number of particle models. However, some possible signatures are possible to discriminate this model from others. In particular, important indications will come out from the observation of the acoustic peaks of the CBR [2 $\left.{ }^{2} \overline{6}\right]$. An analysis of the peaks produced by the decays that we analyzed here could constitute an important complement to the present work.

\section{Acknowledgments}

We _are grateful to _. - Valle for discussions. The _ work of the author. is supported by an INFN Post-Doc fellowship. This work is partially supported by the EEC TMR network Beyond the Standard Model, contract no. FMRX-CT96-0090.

\section{References}

- - [1]- G.B. Blumenthal, S.M. Faber, J_R.. Primack, L - _ _ and M._Rees,_Nature_ 311 _(1984) 517

[2] M. Davis, G. Efstathiou, C.S. Frenk, and S.D.M. White, Nature 356 (1992) 489; K.B. Fisher. M. Davis, M.A. Strauss, A. Yahir, and J.P. Huchra, Astrophys. J. 402 (1993) 42.

- - [3]_ E_W_ Kolb and _M_S_ Turner, The _Early_ Uni-

' - - - serse _(Addison-Wesley_ Publishing- Company, 1990).

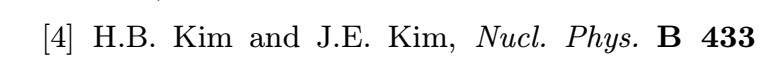

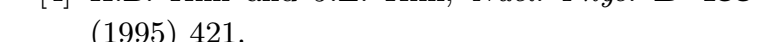

[5] See M.S. Turner, report no. iastro-ph/9901109 for a recent review.

[6] M.S. Turner and J.A. Tyson, report no. astro-ph/9901113:

[7] The Supernova Cosmology Project, S. Perlmutter et al., report no. LBNL-4-1801, astro-ph/9812133; The High- $z$ Supernova Search Team, A.G. Riess et al., Astron. J. 116 (1998) 1009.

[8] J. Bond and G. Efstathiou, Phys. Lett. B 265; - - $(1991) 245$ [9] M. White, G. Gelmini, and J. Silk, 'Phys. Rev.' i:--D-51 (-1995)-2669.

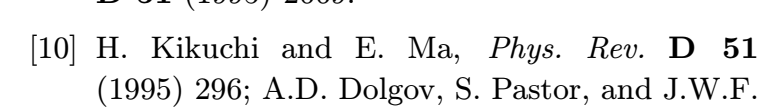
Valle, report no. FTUV/95-14, IFIC/95-14, astro-ph/9506011
[11] A. Masiero, D. Montanino, and A. Peloso, report no. SISSA-15-99-EP, hep-ph/9902380!

[12] J. Gratsias, R.J. Scherrer, and D.N. Spergel, Phys. Lett. B 262 (1991) 298.

[13] E. Lisi, S. Sarkar, and F.L. Villante, report No. INFN-FE-17-98, hep-ph/9901404.

[14] E. Holtmann, M. Kawasaki, K. Kohri, and T. Moroi, report no. LBNL-41826, hep-ph/9805405.

[15] V. Berezinsky, A.S. Joshipura, and J.W.F. Valle, 'Phys. Rev. D.57 (1998) 147.'.

[16] J. Ellis, J.S. Hagelin, D.V. Nanopoulos, K. Olive, and M. Srednicki, №cl. Phys._B 238 $(198 \overline{4}) \overline{453}$.

[17] G. Bhattacharyya, in the proceedings of the Workshop, on Physics Beyond the Standard Model: Beyond the Desert: Accelerator and Nonaccelerator Approach, Tegerneese, Germany, 1997, Ed. by H.V. KlapdorKleingrothaus, Bristol, IOP, 1998, p. 194, hep-ph/9709395.

[18] R.D. Peccei and H.R. Quinn, 'Phys. Rev. D $\mathbf{3} \mathbf{8}_{1}^{\prime}$ (1977) $14 \overline{4} 0$; iPhys. Rev. D $\mathbf{1} \mathbf{6}(1977)$ 1791.

[19] J. Nieves, 'Phys. Lett. B_174 (1986)_411'.

[20] K. Rajagopal, M.S. Turner, and F. Wilczek, Nucl. Phys. B $\mathbf{3} \overline{5} \mathbf{8}(\overline{1991)} 4 \overline{4} 7$.

[21] Y. Chikashige, R.N. Mohapatra, and R.D. Peccei, Phys. Lett. B 98 (1981) 265; 'Phys. Rev.' Lett. 45 (1980) 1926; G.B. Gelmini and M. Roncadelli, 'Phys. Lett. B 99 (1981) 411. H. Georgi, S.L. Glashow, and S. Nussinov, 'Nucl. Phys. B. $193(1981) 297$.

[22] M. Gell-Mann, P. Ramond, and R. Slansky, in the proceedings of Supergravity, Stony Brooke, New York, 1979, ed. by P. Niuwenhuizen and D. Freedman (Amsterdam, NorthHolland, 1979), p. 315; T. Yanagida, in the proceedings of the Workshop on Unified Theories and the Baryon Number of the Universe, Tsukuba,Ibaraki, Japan, 1979, ed. by O. Sawada and A. Sugamoto (KEK report 79-18, Tsukuba, Japan, 1979), p. 95.

[23] G.G. Raffelt Stars as Laboratories for Fundamental Physics (The University of Chicago Press, 1996).

[24] J. Schechter and J.W.F. Valle, Phys. Rev. D 25: (1982) $7 \overline{7}$ !.

[25] P. Fayet, 'Phys. Lett. B_175 (1986)

[26] E. Pierpaoli and S.A. Bonometto, report no. SISSA-55-98-A, 'astro-ph/9806037' 